\title{
MASSIVE BINARIES - EARLY EVOLUTIONARY STAGES
}

\author{
J. B. H U TCHINGS \\ Dominion Astrophysical Observatory, Victoria BC, Canada
}

\begin{abstract}
Fundamental data on $O B$ supergiants and binary systems are presented. Mass loss is reviewed, and its correlation with close binary membership. The evolution of massive binaries is discussed and several classes of observed binaries compared with the theoretical picture. Individual systems of significance are pointed out and the chief problems in massive binary research are indicated.
\end{abstract}

\section{General Description}

The discovery of the supergiant $\mathrm{X}$-ray binaries has focussed a great deal of interest and effort on the study of early-type massive stars, both single and double. To understand the mechanism of X-ray emission it is necessary to know the evolutionary state of the system, its probable history, and the physical interaction between the stars. My purpose is to give a picture of our current observationally-based understanding of the most massive stars.

Our knowledge of OB supergiant binaries is incomplete. The reasons are several. (1) The most massive stars lose mass by a stellar wind at rates that may reduce their masses by a factor of two during their early post-main-sequence lifetimes (Hutchings, 1976). Evolutionary models and mass-loss models have yet to be agreed upon for single stars. The evolution of binaries is one step further away. (2) The spectroscopic effects of mass loss and intrinsic spectrum variations make the detection of binaries difficult and determination of their orbits uncertain. These difficulties are worst for systems with a low mass component. (3) Spectroscopic and photometric effects of mass-exchange in massive binaries are frequently present and prevent information on the underlying stars from being obtained with confidence.

In what follows, therefore, it is worth bearing in mind that what we know is learned through these natural filters.

\section{Masses and Mass Loss}

I shall first present a general picture of the masses of early type stars. The basic data are from binary systems and I have tried to select these which are well behaved or well understood (Hutchings, 1975b). This leaves us with only a few numbers but they seem to form a reasonable pattern (see Tables I, II, Figure 1). The radii are less certain but are tentatively drawn in. The picture is considerably improved by the agreement obtained with single stars whose distance and hence $M_{\mathrm{Bol}}$ is known (a) from cluster memberships (Conti and Burnichon 1975), Figure 1; (b) from $\mathrm{H} \gamma$ or other luminosity indicators (Hutchings 1976), Figure 2 and (c) from the Magellanic Clouds (MC's), Figure 2. The evolutionary tracks are of course for stars of constant mass, but the agreement between workers seems satisfactory and fits well with the binary masses.

This leads to a further aspect of these studies - the mass loss. The distribution of the brightest MC and galactic stars suggests a loss of mass from stars of $60 M_{\odot}$ or more of some $50 \%$ in the upper post-main-sequence evolution. This, by interpolation between 
TABLE I

SB2 binaries with eclipses

\begin{tabular}{|c|c|c|c|c|c|c|c|c|}
\hline Star & & Spectra & $i$ & $M_{V}$ & $M_{\mathrm{Bol}}$ & $M / M_{\odot}$ & $R / R_{\odot}$ & Ref. \\
\hline $\begin{array}{l}\text { HD } \\
34333\end{array}$ & $\begin{array}{l}\text { Other } \\
\text { EO Aur }\end{array}$ & B3 $\times 2$ & $64^{\circ}$ & -4.0 & $\begin{array}{l}-5.8 \\
-5.3\end{array}$ & $\begin{array}{l}27 \\
27\end{array}$ & $\begin{array}{l}16 \\
13\end{array}$ & $1,6,8$ \\
\hline 36486 & $\delta$ Ori A & $09.511+B 1$ & $67^{\circ}$ & -5.8 & $\begin{array}{l}-8.5 \\
-5.5\end{array}$ & $\begin{array}{l}28 \\
10\end{array}$ & $\begin{array}{r}19 \\
9\end{array}$ & $1,2,8$ \\
\hline 198846 & Y Cyg & $09.5 \mathrm{IV} \times 2$ & $86^{\circ}$ & -4.5 & $\begin{array}{l}-6.9 \\
-6.9\end{array}$ & $\begin{array}{l}18 \\
18\end{array}$ & $\begin{array}{l}11 \\
11\end{array}$ & $1,2,8$ \\
\hline 227696 & V453 Cyg & B0.5III $\times 2$ & $78^{\circ}$ & -4.7 & $\begin{array}{l}-7.0 \\
-6.5\end{array}$ & $\begin{array}{l}18 \\
14\end{array}$ & $\begin{array}{l}13 \\
11\end{array}$ & $1,2,8$ \\
\hline 193611 & V478 Cyg & $\mathrm{B} 0.5 \mathrm{~V} \times 2$ & $82^{\circ}$ & -4.3 & $\begin{array}{l}-6.4 \\
-6.4\end{array}$ & $\begin{array}{l}15 \\
15\end{array}$ & $\begin{array}{l}11 \\
11\end{array}$ & $1,6,7,8$ \\
\hline 216014 & AH Cep & B0 $n \times 2$ & $63^{\circ}$ & -4.0 & $\begin{array}{l}-6.3 \\
-6.3\end{array}$ & $\begin{array}{l}16 \\
14\end{array}$ & $\begin{array}{l}7 \\
7\end{array}$ & $1,2,8$ \\
\hline 151890 & $\mu^{1}$ Sco & $\mathrm{B} 1.5 \mathrm{~V}+\mathrm{B} 6$ & $60^{\circ}$ & -4.3 & $\begin{array}{l}-6.0 \\
-4.7\end{array}$ & $\begin{array}{r}14 \\
9\end{array}$ & $\begin{array}{l}5 \\
4\end{array}$ & $1,2,8$ \\
\hline 187879 & V380 Cyg & $\mathrm{B} 1.5+\mathrm{B} 3$ & $76^{\circ}$ & -5.1 & $\begin{array}{l}-7.1 \\
-4.1\end{array}$ & $\begin{array}{r}14 \\
8\end{array}$ & $\begin{array}{l}8 \\
2\end{array}$ & $1,2,8$ \\
\hline 163181 & V453 Sco & $\mathrm{B} 0 \mathrm{I}+\mathrm{O} 8$ & $90^{\circ}$ & -7.0 & $\begin{array}{l}-9.9 \\
-7.0\end{array}$ & $\begin{array}{l}13(\mathrm{U}) \\
22\end{array}$ & $\begin{array}{r}23 \\
9\end{array}$ & 3 \\
\hline 218066 & CW Cep & $\mathrm{B} 1.5 \mathrm{~V} \times 2$ & $82^{\circ}$ & -4.5 & $\begin{array}{l}-6.3 \\
-5.3\end{array}$ & $\begin{array}{l}10 \\
10\end{array}$ & $\begin{array}{l}6 \\
4\end{array}$ & $1,2,8$ \\
\hline 33357 & SX Aur & B3. $5 \times 2$ & $80^{\circ}$ & -3.5 & $\begin{array}{l}-4.5 \\
-3.5\end{array}$ & $\begin{array}{r}11 \\
6\end{array}$ & $\begin{array}{l}5 \\
3.5\end{array}$ & 1,8 \\
\hline 32419 & BF Aur & B5 $\times 2$ & $70^{\circ}$ & -2.0 & $\begin{array}{l}-3.0 \\
-3.0\end{array}$ & $\begin{array}{l}5 \\
5\end{array}$ & $\begin{array}{l}5 \\
5\end{array}$ & 6 \\
\hline & MR Cyg & $\mathrm{B} 3+\mathrm{B} 9$ & $86^{\circ}$ & -2.0 & $\begin{array}{l}-3.5 \\
-0.6\end{array}$ & $\begin{array}{l}4.4 \\
2.5\end{array}$ & $\begin{array}{l}3.9 \\
3.2\end{array}$ & 4,5 \\
\hline 173787 & V356 Sgr & $\mathrm{B} 3 \mathrm{~V}+\mathrm{A} 2 \mathrm{II}$ & $90^{\circ}$ & -3.5 & $\begin{array}{l}-4.5 \\
-4.3\end{array}$ & $\begin{array}{l}12 \\
5(U)\end{array}$ & $\begin{array}{r}5 \\
13\end{array}$ & $1,2,8$ \\
\hline
\end{tabular}

(U) denotes undermassive star

Other systems not included because of complications: V382 Cyg, V444 Cyg, 5 Pic, DH Cep, UW CMa, V Pup, V470 Cyg, HD 47129.

References: (1) Batten, 1968; (2) Koch et al., 1970; (3) Hutchings, 1976; (4) Hill and Hutchings, 1973a; (5) Hill and Hutchings, 1973b; (6) Mammano et al., 1974; (7) Gaposchkin, 1949; (8) Stothers, 1972.

models suggests rates of $\sim 10^{-5} M_{\odot} \mathrm{yr}^{-1}$ from these stars. Other studies and models of stellar winds tend to support these rates (e.g. Castor et al., 1975).

A discussion of stellar winds is not directly relevant to our topic today, but note that there is evidence that the mass-loss rate is enhanced by the presence of a close companion, and in some cases (HD 108, 163181, 187399) the mass loss from the primary is phase correlated. Table III shows the figures from a recent survey of my own, and the implications are clear. We must examine these stars and others like it carefully for evidence of companions.

\section{Evolutionary States of Systems}

I would now like to turn to an examination of massive binaries in different evolutionary states and point to examples and problems which we know of. Table IV shows the way I have divided them and Table $\mathrm{V}$ gives some deduced quantities for examples of each kind. 
TABLE II

SB2 Binaries without eclipses

\begin{tabular}{|c|c|c|c|c|c|c|c|c|}
\hline & Star. & Spectra & $i$ & $M_{V}$ & $M_{\mathrm{Bol}}$ & $M$ & $R$ & Ref. \\
\hline \multicolumn{9}{|c|}{ (1) $i$ derived from assumed $M$} \\
\hline & 191201 & $095 \mathrm{III} \times 2$ & $50^{\circ}$ & -6.5 & $\begin{array}{l}-9.1 \\
-9.1\end{array}$ & $\begin{array}{l}35 \\
35\end{array}$ & $\begin{array}{l}\leqslant 23 \\
\leqslant 23\end{array}$ & 4 \\
\hline & 159176 & $07 \times 2$ & $48^{\circ}$ & -6.0 & $\begin{array}{l}-8.5 \\
-8.5\end{array}$ & $\begin{array}{l}27 \\
26\end{array}$ & $\begin{array}{l}\leqslant 13 \\
\leqslant 13\end{array}$ & 3 \\
\hline & 152218 & O9IV & $70^{\circ}$ & -5.2 & $\begin{array}{l}-8.1 \\
-7.4\end{array}$ & $\begin{array}{l}22 \\
18\end{array}$ & $\begin{array}{l}\leqslant 18 \\
\leqslant 16\end{array}$ & 1 \\
\hline & 152248 & O8f & $55^{\circ}$ & -6.5 & $\begin{array}{l}-9.2 \\
-9.2\end{array}$ & $\begin{array}{l}45 \\
45\end{array}$ & $\begin{array}{l}\leqslant 22 \\
\leqslant 22\end{array}$ & 1 \\
\hline & 206267 & $06+09$ & $50^{\circ}$ & -5.7 & $\begin{array}{l}-9.0 \\
-7.0\end{array}$ & $\begin{array}{l}45 \\
15\end{array}$ & $\begin{array}{l}\leqslant 17 \\
\leqslant 9\end{array}$ & 2 \\
\hline CC Cas & 19820 & $08+09$ & $65^{\circ}$ & -3.9 & $\begin{array}{l}-8.0 \\
-6.3\end{array}$ & $\begin{array}{l}25 \\
12\end{array}$ & $\begin{array}{r}\leqslant 13 \\
\leqslant 9\end{array}$ & 4 \\
\hline \multicolumn{9}{|c|}{ (2) SB2 ellipsoidal } \\
\hline A0 Cas & 1337 & $09+08$ & $50^{\circ}$ & -4.4 & $\begin{array}{l}-6.9 \\
-6.9\end{array}$ & $\begin{array}{l}18 \\
23\end{array}$ & $\begin{array}{l}12 \\
10\end{array}$ & 5 \\
\hline$\psi$ Ori & 35715 & $\mathrm{~B} 0.5+\mathrm{B} 2$ & $58^{\circ}$ & -3.8 & $\begin{array}{l}-6.0 \\
-5.0\end{array}$ & $\begin{array}{r}14 \\
9\end{array}$ & $\begin{array}{l}6 \\
5\end{array}$ & 5 \\
\hline \multicolumn{9}{|c|}{ (3) SB1 eclipsing (X-ray) } \\
\hline & 153919 & O6f & $90^{\circ}$ & -6.6 & -10.3 & $27(\mathrm{U})$ & 20 & 6 \\
\hline & 77581 & B0I & $90^{\circ}$ & -7.3 & -10.3 & 22 (U?) & 35 & 6,7 \\
\hline & Cen X-3 & 06 & $90^{\circ}$ & - & - & $17(\mathrm{U})$ & 12 & 6 \\
\hline & HZ Her & A0 & $90^{\circ}$ & - & - & 2.0 & 1.7 & 6 \\
\hline
\end{tabular}

References: (1) Hill et al., 1974; (2) Crampton and Redman, 1975; (3) Conti et al., 1975; (4) Batten, 1968; (5) Hutchings and Hill, 1971; (6) Hutchings, 1975a; (7) Rappoport et al., 1976.

It is appropriate to give a short summary of different paths of evolution for massive binaries, as they are presently understood or proposed, in order to see how the observed types of binaries fit into or make up the general picture. The initial mass ratios are probably not far from unity (i.e. $\lesssim 4$ ) as the radiation and stellar wind from the more rapidly evolving, more massive star will tend to destroy a low mass companion while it is still in early stages of condensation. Thereafter follow the well known cases of mass exchange depending on the stage at which the more massive star fills its roche lobe. Because mass loss from the initial primary may be considerable, it does not follow that less evolved mass-accreting star be less massive. Further, mass exchange is generally very rapid and does not necessarily result in a great lowering of luminosity. It may, however, expose CNO processed material and lead to the observation of abundance anomalies. This is thought to be the origin of the WN and WC stars, and may be connected with the 'CNO' stars. Finally, after the initial primary has completed its evolution it may end up as a (much?) less massive accreting condensed object as the initial secondary evolves. Such systems include the well known X-ray binaries.

Table IV, is not a complete table, but one which gives examples of the different types of binary. In the pre-interaction group I would point out A0 Cas as being of interest. The model derived for this system (Hutchings and Hill, 1971) suggests that the stars are close to undergoing mass exchange, as one of the components is somewhat underluminous 


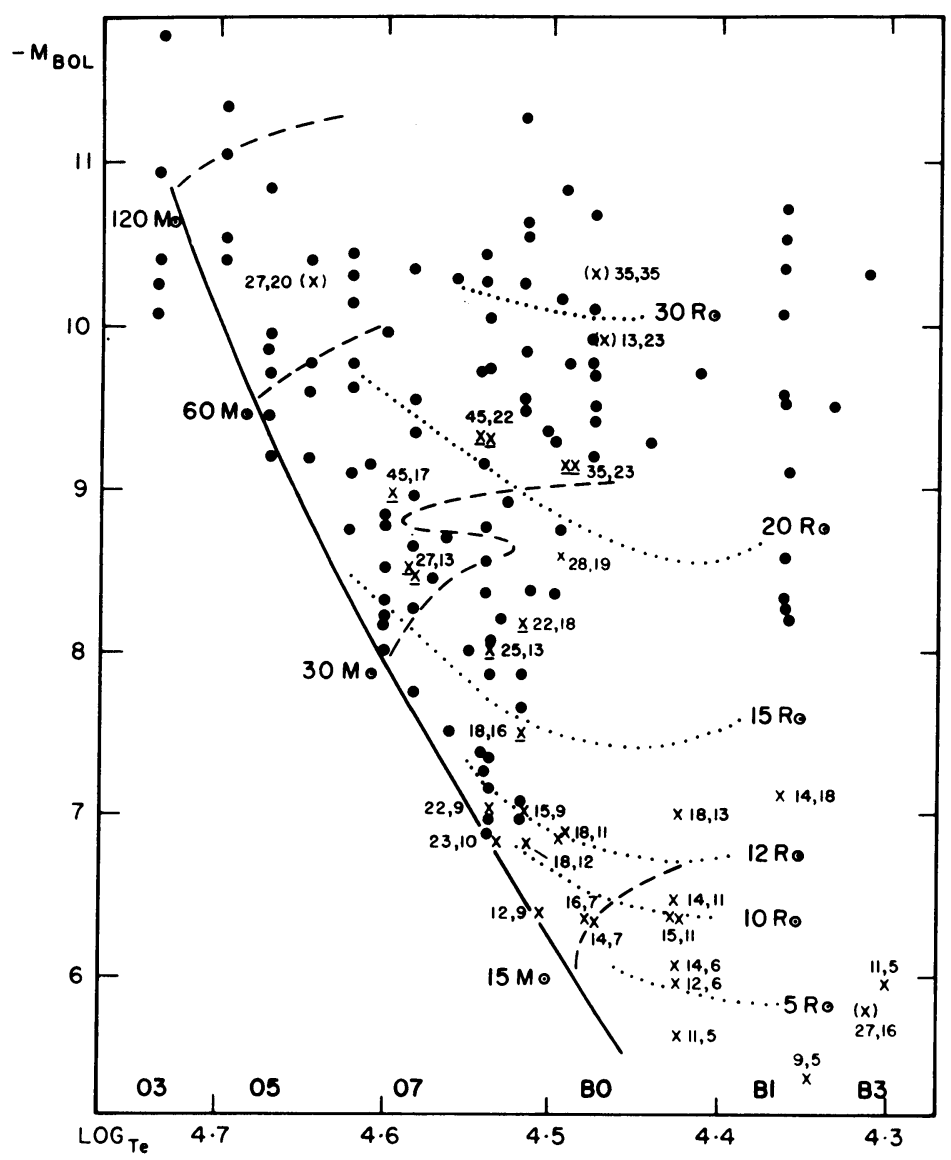

Fig. 1. Theoretical H-R diagram with individual OB stars. Dots: $O$ stars with known distances. Crosses: binaries of known mass and luminosity. Approximate stellar radius contours drawn in.

for its radius and overluminous for its spectral type, if it were a main sequence object. This system is one of the few ellipsoidal variables studied in any detail and more such investigations need to be made.

In the unequal mass column, HD 206267 is worthy of a brief comment. It was a candidate star for an X-ray source, until detailed spectroscopy (Crampton and Redman, $1975)$ revealed the presence of a normal unevolved companion of lower mass. The existence and statistics of such binaries are still largely unknown and an assessment of the numbers of stars with normal and collapsed companions is important to our proper understanding of their evolutionary processes.

The next column isolates stars (other than W-R stars, which I do not intend to cover) with undermassive primaries. Two of the four given also show abundance anomalies. At present such objects seem to be rare and anomalous but until our statistics are better it is not clear whether mass exchange in binaries is the cause of these phenomena. $\beta$ Lyr appears to be in the stage of rapid mass exchange and the primary is not strongly over- 


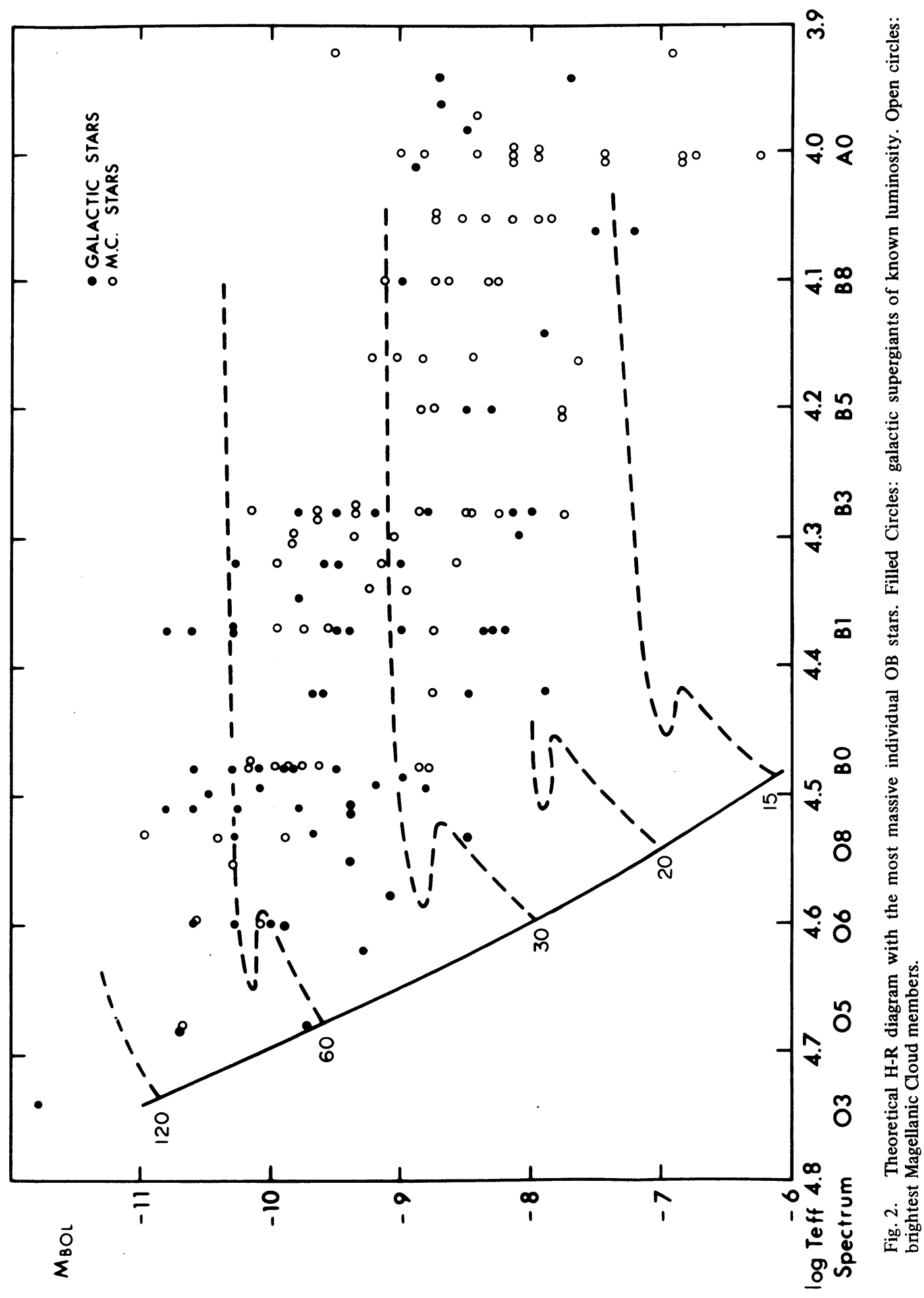


TABLE III

Mass-loss rates (in $10^{-7} M_{\odot} \mathrm{yr}^{-1}$ ) for selected groups of stars

\begin{tabular}{|c|c|c|c|c|c|c|c|c|}
\hline \multicolumn{3}{|c|}{ Mass-loss rate $\geqslant 100$ or high for $M_{\mathrm{Bol}}$} & \multicolumn{3}{|c|}{ Known binary stars } & \multicolumn{3}{|c|}{ Variable velocity stars } \\
\hline Star HD & Remark & $-\dot{m}$ & Star HD & Remark & $-\dot{m}$ & Star HD & Remark & $-\dot{m}$ \\
\hline $\begin{array}{l}108 \\
105056 \\
152236 \\
152408 \\
152667 \\
153919 \\
163181 \\
169454 \\
190603 \\
193237 \\
226868\end{array}$ & $\begin{array}{l}\text { binary } \\
\text { variable vel. } \\
\text { variable vel. } \\
\text { variable vel. } \\
\text { binary } \\
\text { binary } \\
\text { binary } \\
\text { variable vel. } \\
\text { variable vel. } \\
\text { variable vel. } \\
\text { binary }\end{array}$ & $\begin{array}{r}1000 \\
300 \\
500 \\
900 \\
270 \\
150 \\
100 \\
100 \\
500 \\
3500 \\
25\end{array}$ & $\begin{array}{l}108 \\
36486 \\
57060 \\
77581 \\
148937 \\
152667 \\
153919 \\
163181 \\
166937 \\
226868\end{array}$ & $\begin{array}{l}\text { high } \\
\text { low luminosity }\end{array}$ & $\begin{array}{r}1000 \\
1 \\
85 \\
70 \\
80 \\
270 \\
150 \\
100 \\
50 \\
25\end{array}$ & $\begin{array}{l}105056 \\
149404 \\
150958 \\
152236 \\
152408 \\
169454 \\
178129 \\
190603 \\
193237\end{array}$ & $\begin{array}{l}\text { high } \\
\text { high } \\
\text { high } \\
\text { high } \\
\text { high } \\
\text { high }\end{array}$ & $\begin{array}{r}300 \\
35 \\
65 \\
500 \\
900 \\
100 \\
40 \\
500 \\
3500\end{array}$ \\
\hline
\end{tabular}

Mean $-\dot{m}$ for all stars (except 193237) 91

all known binary stars 183

all variable velocity stars

(except 193237) 310

luminous or peculiar in abundance. HD 163181 has definite oxygen weakness and is undermassive by more than a factor of two (Hutchings, 1975b). Mass loss from the primary is high for a star of its luminosity, but the system is not in the rapid mass exchange phase we see in $\beta$ Lyr. Other presumably post mass exchange systems do not show these anomalies. It may be that few stars exchange sufficient mass, or that composition anomalies are seen only for a short time after mass exchange. There is a class of 'CNO' stars which show abundance anomalies. A review of these is given by Jaschek and Jaschek (1974) but their objects appear to me to be a mixture, including some quite normal stars. I have investigated five of their objects for binary membership and find that two are clearly not binaries, two may be, and one is. However, in four out of eight of these stars, I find no spectral anomalies of the type claimed.

Two more undermassive primaries - HD 153919 and Cen X-3 - are X-ray source companions (e.g. Hutchings, 1975c). Data on the former show no clear abundance anomalies and the mass is in some doubt. There is no doubt that the mass of Cen X-3 is

TABLE IV

Massive binaries of early type

\begin{tabular}{|c|c|c|c|c|c|}
\hline \multicolumn{2}{|c|}{ Pre mass-exchange } & \multicolumn{4}{|l|}{ Mass-exchange } \\
\hline $\begin{array}{l}\text { Equal } \\
\text { mass }\end{array}$ & $\begin{array}{l}\text { Unequal } \\
\text { mass }\end{array}$ & $\begin{array}{l}\text { Undermassive } \\
\text { primary }\end{array}$ & $\begin{array}{l}\text { Very low mass } \\
\text { companion }\end{array}$ & $\begin{array}{l}\text { High mass } \\
\text { companion }\end{array}$ & Others \\
\hline $\begin{array}{l}\text { A0 Cas } \\
\text { E0 Aur } \\
\text { etc. }\end{array}$ & $\begin{array}{l}206267 \\
\delta \text { Ori A } \\
\text { etc. }\end{array}$ & $\begin{array}{l}\beta \text { Lyr } \\
163181 \\
153919 \\
\text { Cen X-3 } \\
\\
+40^{\circ} 4220\end{array}$ & $\begin{array}{l}108 \\
148937 \\
77581 \\
\text { SMC X-1 } \\
153919 \\
\text { Cen X-3 } \\
152667\end{array}$ & $\begin{array}{l}226868 \\
187399 \\
173219 \\
72754 \\
\text { X Per? } \\
47129 \\
\beta \text { Lyr }\end{array}$ & $\begin{array}{l}\text { W-R stars? } \\
\text { VV Cep stars } \\
\text { Symbiotic stars }\end{array}$ \\
\hline
\end{tabular}


low for its spectral type, but spectroscopic data of this faint star are poor. Other X-ray source primaries, which are B stars, are not obviously undermassive.

We now turn to systems with low mass companions. When the mass ratio is 10 or more, it seems very probable that the invisible companion is an evolved star of some sort, and most of the X-ray binaries are in this category. I would also particularly draw attention to HD 108 and 148937 which are extreme Of stars, very similar to HD 153919. They both have orbital motions which indicate secondary companions of $1-3 M_{\odot}$ (Hutchings, 1975d: Hutchings and Conti, 1976). HD 148937 is centred in some nebulosity and has been suggested (Pisimis, 1974) as a supernova remnant. Mass accretion rates for these two secondaries are probably insufficient to power a detectable X-ray source. Systems of this nature - and there are several more candidates under investigation - are of importance in understanding this stage of evolution.

The final group of objects is one for which I see no clear evolutionary explanation and it may be that the group contains several different types of object. They all have secondary stars which are more massive and less luminous than the primary. The masses implied suggest that they are either blanketed early type stars or black holes. Recent evidence from UV emission lines in $\beta$ Lyr tends to favour the black hole model for its secondary. HD 187399 and 173219 both have mass functions that indicate companions of greater mass than the primaries, for all reasonable primary masses. Both primaries are losing mass at fairly high rates. If X Per is a binary (as suggested by Hutchings et al., $1974)$ then its companion is very massive $\left(40 M_{\odot}\right.$ ?), very well separated, and probably the weak X-ray source $2 \mathrm{U} 0352+30$. In this case it is possible the companion completed its evolution without losing most of its mass, but in the other systems we must propose that the stars were initially much more widely separated. The secondary of Plaskett's star (HD 47129) has long been thought to be a massive underluminous OB star, but recent high dispersion spectroscopy suggests that the secondary spectrum may be partly caused by gas streams (Hutchings and Cowley, 1976). Again for all reasonable primary masses, the secondary is more massive.

TABLE V

\begin{tabular}{lllllll}
\hline System & & \multicolumn{3}{c}{ Some post mass-exchange system parameters } & \\
\cline { 3 - 6 } & & $M_{1}$ & $M_{2}$ & $f(m)$ & $i$ & \\
\hline B Lyr & B8p & $4 ?$ & $16 ?$ & 8.5 & $\sim 90^{\circ}$ & \\
163181 & BOIa & 13 & 22 & 8.8 & $\sim 75^{\circ}$ & undermassive \\
153919 & O6f & 27 & 1.5 & 0.0027 & $\sim 90^{\circ}$ & primary \\
Cen X-3 & O6 & 17 & 0.7 & - & $\sim 90^{\circ}$ & \\
$+40^{\circ} 4220$ & O6 + O7 & 15 & 60 & 31 & $\sim 70^{\circ}$ & \\
108 & & & & & \\
148937 & O8f & $60 ?$ & $\sim 2$ & .0004 & $60^{\circ} ?$ & \\
77581 & O8f & $60 ?$ & $\sim 3$ & .004 & $60^{\circ} ?$ & low mass \\
& B0I & 22 & 0.6 & .007 & $90^{\circ}$ & secondary \\
Cyg X-1 & B0I & 30 & 16 & 0.2 & $30^{\circ}$ & \\
187399 & B8e & 3 & 6 & 2.6 & $\sim 90^{\circ}$ & \\
173219 & B0e & 12 & 24 & 11 & $\sim 90^{\circ} ?$ & high mass \\
72754 & Bp & 23 & 29 & 9 & $\sim 90^{\circ}$ & secondary \\
X Per & B0e & 20 & 40 & 18 & $60^{\circ} ?$ & \\
47129 & O9e & $60 ?$ & $60 ?$ & 13 & $70^{\circ} ?$ & \\
\hline
\end{tabular}




\section{Problems}

We are left, as in all active fields of research, with a number of important general questions. These seem to me to be the following. We are not yet clear as to the evolutionary effects of mass loss or mass exchange in massive star systems. Many arguments suggest that they are large and important - e.g. the short period of systems like Cen X-3 (MeyerHofmeister, 1974). The CNO abundance anomalies - are they fact or fiction, and are they connected with rapid or extensive mass transfer? Are they correlated with undermassive primary stars? How many massive binaries are there and how many contain collapsed components? Do we observe the right number of X-ray sources in the galaxy? Finally, what are the mysterious unseen massive companions to some $O$ and $B$ stars? Critical observations of high quality are needed to answer many of these questions.

\section{References}

Batten, A. H.: 1968, Astron. J. 73, 551.

Castor, J. I., Abbott, D. C., and Klein, R. I.: 1975, Astrophys. J. 195, 157.

Conti, P. S. and Burnichon, M. L.: 1975, Astron. Astrophys. 38, 46.7.

Conti, P. S., Cowley, A. P., and Johnson, G. B.: 1975, preprint.

Crampton, D. and Redman, R. O.: 1975, Astron. J. 80, 454.

Gaposhkin, S.: 1949, Astron. J. 54, 128.

Hill, G., Crawford, D. L., and Barnes, J. V.: 1974, Astron. J. 79, 1271.

Hill, G. and Hutchings, J. B.: 1973a, Astrophys. Space Sci. 20, 123.

Hill, G. and Hutchings, J. B.: 1973b, Astron. Astrophys. 23, 357.

Hutchings, J. B.: 1975a, Publ. Astron. Soc. Pacific 87, 529.

Hutchings, J. B.: 1975b, Publ. Astron. Soc. Pacific 87, 245.

Hutchings, J. B.: 1975c, Astrophys. J. 201, 413.

Hutchings, J. B.: 1975d, Astrophys. J. 200, 122.

Hu tchings, J. B.: 1976, Astrophys. J. 203, 438.

Hutchings, J. B. and Conti, P. S.: 1976, Astrophys. J. (in press).

Hutchings, J. B. and Cowley, A. P.: 1976, Astrophys. J. (in press).

Hutchings, J. B., Crampton, D., and Redman, R. O.: 1974, Monthly Notices Roy. Astron. Soc. 170, 313.

Hutchings, J. B. and Hill, G.: 1971, Astrophys. J. 167, 137.

Jaschek, M. and Jaschek, C.: 1974, Astron. Astrophys. 36, 401.

Koch, R. H., Plavec, M., and Wood, F. B.: 1970, Univ. of Penns. Publ. Vol. X.

Mammano, A., Margoni, R., and Stagni, R.: 1974, Astron. Astrophys. 35, 143.

Meyer-Hofmeister, E.: 1974, Astron. Astrophys. 36, 261.

Pisimis, P.: 1974, Rev. Mexico Astron. Astrophys. 1, 45.

Rappoport, S., Joss, P. C., McClintock, J. E.: 1976, Astrophys. J. (in press).

Stothers, R.: 1972, Astrophys. J. 175, 431.

\section{DISCUSSION}

Van den Heuvel: I do not agree with your assertion that the UV emission lines would require.a special collapsed object in $\beta$ Lyr. All UV emission lines are collisionally excited, and can be explained like lines in the solar corona by an acoustically heated corona, although around a disk-like star in this case. Such a disk will have large turbulence, and hence, a large acoustic energy flux, and, consequently, a hot corona.

Hutchings: I think the point can be argued either way. You would have to produce some numbers to show that such a large line flux (larger than the continuum flux) can be generated in this way.

Bolton: There are many more OB stars than has been previously recognised. In the HD catalogue many OB stars are classified as B8 or A0 because of the interstellar $\mathrm{Ca}$ II lines. The much neglected eclipsing binary DR Vul has been classified as B8 when in fact it is about B1.

Paczynski: I believe there is no particular mystery about the binary systems with invisible massive secondaries. In the case of $\beta$ Lyr Dr Ziołkowski produced theoretical evolutionary models that explain the observations very well. 
Ostriker: Classifying stars on the H-R diagram, you find that massive binary components have an anomolously high mass loss rate. If you classify the stars by effective temperature and surface gravity, are the binaries still peculiar? Rotation and the tidal force from the companion reduce surface gravity. Perhaps mass loss has an acoustic wave origin in part (as in the solar wind) rather than being purely due to radiation pressure (from which one would not expect a strong dependence on surface gravity).

Hutchings: You do find a surface gravity dependence in the sense that primaries of close binaries in the sample generally are tidally distorted and have lower surface gravity in some parts of their surface. In addition, in some binaries the mass loss rate is phase dependent in the sense that it is enhanced either at periastron in a non-circular orbit, or in the direction of the companion where tidal distortion is large.

Van den Heuvel: How sure can one be about the place in X-ray systems where the He II 4686 line comes from? It was not clear to me whether you assumed it to have come from the star or from the X-ray source.

Hutchings: We assume the line emission to arise close to the X-ray emitter. We can exclude (on the basis of phasing of the light curve, lack of variation of emission lines intensity, and lack of strong heating effect) models in which the emission originates on the non-X-ray stars, or in the stream. 